Submitted to Phys. Rev. Lett.

\title{
The pair-breaking critical current density of magnesium diboride
}

\author{
Milind N. Kunchur \\ Department of Physics and Astronomy \\ University of South Carolina, Columbia, SC 29208 \\ Sung-Ik Lee and W. N. Kang \\ Department of Physics, \\ Pohang University of Science and Technology \\ Pohang 790-784, Republic of Korea
}

(Dated: October 30, 2018)

\begin{abstract}
The pair-breaking critical current density, $j_{d}$, of magnesium diboride was determined over its entire temperature range by a pulsed dc transport measurement. At fixed low values of current density $j$, the resistive transition temperature $T_{c}$ shifts in the classic $\Delta T_{C}(j) / T_{C}(0) \propto-\left[j / j_{d}(0)\right]^{2 / 3}$ manner, with a projected $j_{d}(0) \approx 2 \times 10^{7} \mathrm{~A} / \mathrm{cm}^{2}$. The directly measured $j_{d}(0)$, from currentvoltage $(I-V)$ curves at different fixed temperatures, has a similar value and the overall temperature dependence $j_{d}(T)$ and magnitude are consistent with Ginzburg-Landau theory.

PACS numbers: $74.25 . \mathrm{Sv}, 74.25 . \mathrm{Fy}, 74.25 . \mathrm{Bt}$
\end{abstract}

\section{INTRODUCTION}

Magnesium diboride $\left(\mathrm{MgB}_{2}\right)$ recently made an impact as a promising new superconductor with a surprisingly high critical temperature for a simple binary compound. This has spurred considerable research activity into investigating the myriad properties associated with its superconducting state. Besides the critical temperature $T_{c}$ and the upper critical field $H_{c 2}$, an intrinsic parameter that sets a fundamental limit to the survival of superconductivity is the pair-breaking (or depairing) critical current density $j_{d}$. We report the first measurement of this important quantity in the $\mathrm{MgB}_{2}$ superconductor, which sets an absolute limit to the maximum current-carrying performance under ideal conditions. This also represents, to our knowledge, the only complete $\left(0 \lesssim T \lesssim T_{c}\right)$ measurement of $j_{d}$ by a direct transport method in any typeII superconductor.

When a superconducting state is formed, charge carriers correlate and condense into a coherent macroscopic quantum state. The formation of this state is governed principally by a competition between four energies: condensation, magnetic-field expulsion, thermal, and kinetic. The order parameter $\Delta$, that describes the extent of condensation and the strength of the superconducting state, is reduced as the temperature $T$, magntetic field $H$, and electric current density $j$ are increased. The boundary in the $T-H-j$ phase space that separates the superconducting and normal states is where $\Delta$ vanishes, and the three parameters attain their critical values $T_{c}(H, j)$, $H_{c 2}(T, j)$, and $j_{d}(T, H)$.

In practice, a superconductor loses its ability to carry resistanceless current long before $j$ reaches $j_{d}$. Any process that causes the phase difference between two points to change with time - such as the motion of flux vortices, phase slip centers in narrow wires, junctions, and fluctuations - can generate a finite voltage and hence resistance. The conventional critical-current density $j_{c}$ marks this onset of dissipation - depending on extrinsic variables such as vortex pinning by defects-and in type-II superconductors can be a few orders of magnitude lower than $j_{d}$. Thus the transport becomes resistive and intensely dissipative long before the thermodynamic limit is reached, tending to mask a direct measurement of $j_{d}$ by sample heating. In this work we use a highly evolved pulsed-current technique (which we have refined over ten years) to reduce heating and obtain $j_{d}(T \rightarrow 0)$ both from (1) a direct measurement of $j$ required to drive the system normal at $T \ll T_{c}$ and (2) from the shift in $T_{c}$ as a function of $j$ near $T \sim T_{c}$.

\section{THEORETICAL BACKGROUND}

A theoretical estimate of $j_{d}$ can be obtained from the Ginzburg-Landau (GL) theory, in which the strength of the superconducting state is expressed through the complex phenomenogical order parameter $\psi=|\psi| e^{i \varphi}$. The superfluid density near $T_{c}$ is proportional to $|\psi|^{2}$ and the free-energy density $f$ of the system (w.r.t. the freeenergy density in the normal state) can be expressed as a power expansion in $|\psi|^{2}$ (In "dirty" superconductorssuperconductors with a high impurity scattering rate the approximate validity of the GL expressions extends down to $T \ll T_{c}$.). In the absence of significant magnetic fields and in situations where the magnitude of the order parameter $|\psi|$ is uniform (either because the dimensions of the sample are small compared to the coherence length or because of the principle of minimum entropy production at high dissipation levels [1]) $f$ can be expressed as [2]

$$
f=\alpha|\psi|^{2}+\frac{\beta}{2}|\psi|^{4}+\frac{1}{2}|\psi|^{2} m^{*} v_{s}^{2} .
$$

$\alpha$ and $\beta$ are negative and positive constants respectively ( $\alpha$ becomes positive above $T_{c}$ ), and the positive third 
term is the kinetic energy density expressed in terms of the superfluid velocity $\boldsymbol{v}_{s}=\frac{\hbar \nabla \varphi}{m^{*}}-\frac{e^{*} \boldsymbol{A}}{c m^{*}}$; where $e^{*}$ and $m^{*}$ are respectively the effective charge and mass of a cooper pair. For zero $v_{s}$, the equilibrium value of $|\psi|^{2}$ that minimizes the free energy (Eq. 11) is $\left|\psi_{\infty}\right|^{2}=-\alpha / \beta$. For a finite $v_{s}$ it becomes

$$
|\psi|^{2}=\left|\psi_{\infty}\right|^{2}\left(1-\frac{m^{*} v_{s}^{2}}{2|\alpha|}\right) .
$$

The corresponding supercurrent density is

$$
j=e^{*}|\psi|^{2} v_{s}=2 e\left|\psi_{\infty}\right|^{2}\left(1-\frac{m^{*} v_{s}^{2}}{2|\alpha|}\right) v_{s} .
$$

The maximum possible value of this expression can now be identified with $j_{d}$ :

$$
j_{d}(T)=2 e\left|\psi_{\infty}\right|^{2} \frac{2}{3}\left(\frac{2 \alpha}{3 m^{*}}\right)^{1 / 2}=\frac{c H_{c}(T)}{3 \sqrt{6} \pi \lambda(T)}
$$

where the GL-theory parameters were replaced by their expressions $\alpha(T)=-\left(e^{* 2} / m^{*} c^{2}\right) H_{c}^{2}(T) \lambda^{2}(T)$ and $\beta(T)=\left(4 \pi e^{* 4} / m^{* 2} c^{4}\right) H_{c}^{2}(T) \lambda^{4}(T)$ in terms of the physically measureable quantities $H_{c}$ (thermodynamic critical field) and $\lambda$ (magnetic penetration depth). The relations $H_{c}(T) \approx H_{c}(0)\left[1-\left(T / T_{c}\right)^{2}\right]$ and $\lambda(T) \approx$ $\lambda(0) / \sqrt{\left[1-\left(T / T_{c}\right)^{4}\right]}$ give

$$
j_{d}(T) \approx j_{d}(0)\left[1-\left(T / T_{c}\right)^{2}\right]^{\frac{3}{2}}\left[1+\left(T / T_{c}\right)^{2}\right]^{\frac{1}{2}}
$$

where

$$
j_{d}(0)=c H_{c}(0) /[3 \sqrt{6} \pi \lambda(0)]
$$

is the zero-temperature depairing current density. Close to $T_{c}$, Eq. 5 reduces to $j_{d}\left(T \approx T_{c}\right) \approx 4 j_{d}(0)\left[1-T / T_{c}\right]^{\frac{3}{2}}$. This can be inverted to give the shift in transition temperature $T_{c}(j)$ at small currents, with the well-known $j^{2 / 3}$ proportionality:

$$
\frac{T_{c}(0)-T_{c}(j)}{T_{c}(0)} \approx\left(\frac{1}{4}\right)^{\frac{2}{3}}\left[\frac{j}{j_{d}(0)}\right]^{\frac{2}{3}} .
$$

(The preceding discussion is based on Refs. [2] and [3].) Note that if heat removal from the sample is ineffective, Joule heating will give an apparent shift $\Delta T_{c} \propto \rho j^{2}$, which is the cube of the intrinsic $\sim j^{2 / 3}$ depairing shift near $T_{c}$, and hence easily distinguishable.

\section{EXPERIMENTAL DETAILS}

The samples are $400 \mathrm{~nm}$ thick films of $\mathrm{MgB}_{2}$ fabricated using a two-step method whose details are described elsewhere 4, 5]. An amorphous boron film was deposited on a (1102) $\mathrm{Al}_{2} \mathrm{O}_{3}$ substrate at room temperature by pulsed-laser ablation. The boron film was then put into a $\mathrm{Nb}$ tube with high-purity $\mathrm{Mg}$ metal (99.9\%) and the $\mathrm{Nb}$ tube was then sealed using an arc furnace in an argon atmosphere. Finally, the heat treatment was carried out at $900^{\circ} \mathrm{C}$ for $30 \mathrm{~min}$. in an evacuated quartz ampoule sealed under high vacuum. X-ray diffraction indicates a highly c-axis-oriented crystal structure normal to the substrate surface with no impurity phases. The films were photolithographically patterned down to narrow bridges. In this paper we show data on three bridges, labelled S, M, and L (for small, medium, and large) with lateral dimensions $2.8 \times 33,3.0 \times 61$, and $9.7 \times 172 \mu \mathrm{m}^{2}$ respectively. The lateral dimensions are uncertain by $\pm 0.7 \mu \mathrm{m}$ and the thickness by $\pm 50 \mathrm{~nm}$.

The electrical transport measurements were made using a pulsed signal source with pulse durations ranging $0.1-4 \mu \mathrm{s}$ and a duty cycle of about $1 \mathrm{ppm}$. From past experience with other films (e.g., $\mathrm{Y}_{1} \mathrm{Ba}_{2} \mathrm{Cu}_{3} \mathrm{O}_{7}$ on $\mathrm{LaAlO}_{3}$ ) we found that micron-wide bridges typically have thermal resistances of order $R_{t h} \sim 1-10 \mathrm{nK} . \mathrm{cm}^{3} / \mathrm{W}$ at microsecond timescales [1, 6, 7]. For the present film-substrate combination, complete information about the thermal constants was not available to calculate $R_{t h}$ from first principles, but we are able to show by other means that sample heating is not appreciable. Further details of the measurement techniques have been published in a previous review article [7] and other recent papers [1], 6]. All measurements were made in zero applied magnetic field and the highest self field of the current $(\sim 300 \mathrm{G})$ is of the order of the lower-critical field $H_{c 1}=185 \mathrm{G}$ [8] .

\section{RESULTS AND ANALYSIS}

Fig. 1(a) shows the resistive transitions at different electric currents $I$ for the medium sample. The inset shows the sample geometry. The horizontal sections of the current leads add a small $(\sim 15 \%)$ series resistance to the actual resistance of the bridge. Because $j$ in these wide regions is negligible, this resistance freezes out at the nominal unshifted $T_{c}$, making the onset seem to not shift. However over the main portions of the curves, there are substantial and relatively parallel shifts induced by current. Fig. If(b) provides a magnified view of the central two-thirds portion of the transitions. The dashed line represents half the normal-state resistance $\left(R_{n}\right)$ of the bridge, which serves as the criterion (resistive-transitionmidpoint) for defining $T_{c}(j)$. Panels (c) and (d) show similar sets of curves for the other two (small and large) samples.

Fig. 2 shows the midpoint $T_{c}$ 's and their corresponding currents (ranging from $10^{-6}$ to $10^{-2} \mathrm{~A}$ ) plotted as $I^{2 / 3}$ (expected for pair-breaking) and as $I^{2}$ (expected for Joule heating). The shifts are closely proportional to $I^{2 / 3}$ rather than to $I^{2}$, showing that heating is not appreciable (the plots for samples $\mathrm{S}$ and $\mathrm{L}$ look similar). The slope $d I^{2 / 3} / d T_{c}(j)$ together with Eq. 7 gives a zerotemperature depairing current value of $257 \mathrm{~mA}$. Dividing this by the cross-sectional area gives a current density of 

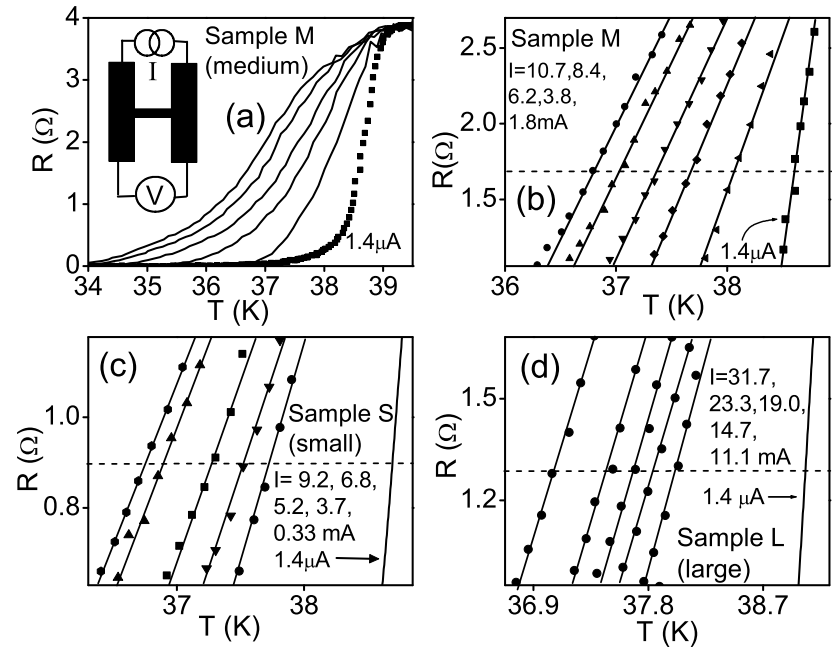

FIG. 1: Resistive transitions of $M g B_{2}$ bridges at different currents (values correspond to curves from left to right.). Panels (a) and (b) show two windows of the same data. The inset in (a) shows the sample geometry and configuration of leads. Panels (b), (c), and (d) show the central main portions of the transitions for three different sized samples. The rightmost curves at $I=1.4 \mu \mathrm{A}$ were measured by with a continuous $D C$ current; the rest used pulsed signals. The dashed lines represents $R=R_{n} / 2$ for each sample.

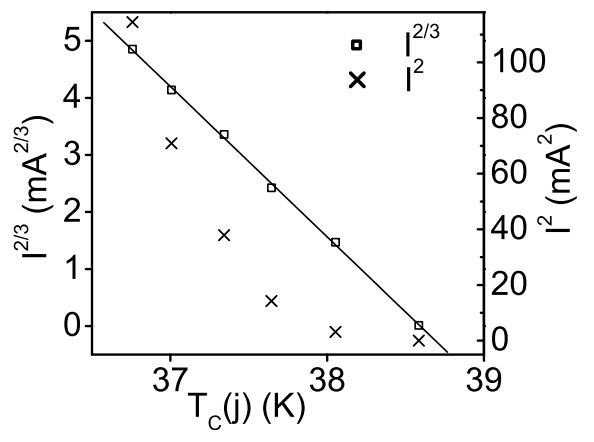

FIG. 2: Shifted transition temperatures at different currents. The two $y$-axes plot the same $T_{c}(j)$ data versus $I^{2 / 3}$ and $I^{2}$, showing adherance to the $I^{2 / 3}$ law for pair breaking rather than the $I^{2}$ law for Joule heating. The linear fit (solid line) to the $I^{2 / 3}$ plot gives $I_{d}(0)=257 \mathrm{~mA}$ (see Eq. 可).

$j_{d}(0)=2.1 \times 10^{7} \mathrm{~A} / \mathrm{cm}^{2}$. The respective values for samples S and L are $j_{d}(0)=2.2 \times 10^{7}$ and $1.8 \times 10^{7} \mathrm{~A} / \mathrm{cm}^{2}$. The three values are consistent within the uncertainities in the sample dimensions, implying a cross-sectionally uniform current density. This is expected for the dissipative state of a superconductor (In the fluctuation region near the $T_{c}(j)$ boundary and during flux motion-when the superconductor is resistive - the current flow becomes macroscopically uniform, as in a normal conductor, due to the principle of minimum entropy production. This has been discussed and verifed elsewhere [1].) and close to the $T_{c}(j)$ boundary where $\lambda$ and $\xi$ (coherence length) diverge.
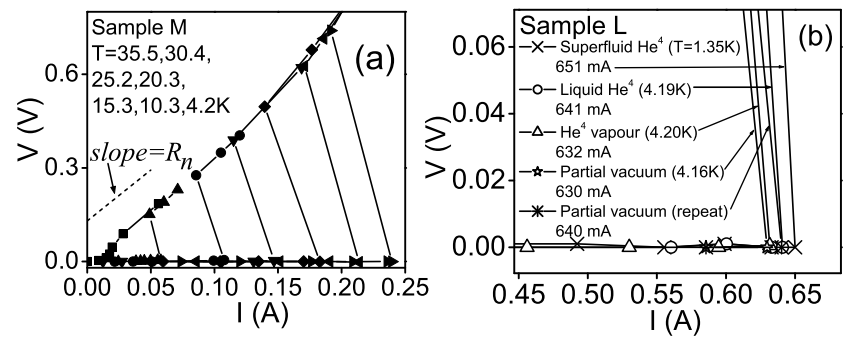

FIG. 3: (a) IV curves for sample $M$ at seven fixed temperatures (listed for curves going from left to right). Beyond $j_{d}$, the voltage jumps to a linear behavior reflecting the resistance of the normal state (slope indicated by the dashed line). (b) $I V$ curves for the largest sample in different thermal environments to evaluate Joule heating.

Fig. 3(a) shows current-voltage $(I V)$ characteristics at various fixed temperatures for sample $\mathrm{M}$ (results for samples $\mathrm{S}$ and $\mathrm{L}$ are similar). As $I$ is increased $V$ remains close to zero until some critical value. Above this it shows Ohmic behavior $V=I R_{n}$. Note that at $T=35.5 \mathrm{~K}$ the transition is gradual, whereas at the lower temperatures it is rather abrupt. This is in part because a type II superconducting phase transition changes from second order to first order at lower temperatures in the presence of a current [3] and possibly because of a thermal component. The "s" shape arises because the external circuit feeding the pulsed signal has a source impedance $R_{s}$ of about $12 \Omega$. Thus when the sample is driven normal, the current will drop discontinuously by the fraction $R_{n} /\left(R_{n}+R_{s}\right) \sim 20 \%$ as observed. Although the amount of Joule heating cannot be directly estimated, its significance can be assessed by measuring the $I V$ curves in different thermal environments. The previous curves in Fig. 3(a) were all measured with the sample in helium vapour. Such measurements were repeated with the samples in superfluid and normal liquid helium, and in vacuum, and Fig. 3(b) shows one such set for the large sample (its lower surface-to-volume ratio gives it the worst thermal resistance). Fig. 3(b) shows no significant systematic influence of the environment on the observed value of $j_{d}$, which would not be the case if Joule heating were a serious problem [9].

From such $I V$ characteristics measured at the lowest temperature $(1.5 \mathrm{~K})$ in superfluid helium, the current required to drive the sample normal provides a direct lower bound on $j_{d}(T \approx 0)$ (This lower bound will equal $j_{d}$ in the case of uniform current flow. We return to this point again later.). For the three samples $\mathrm{S}, \mathrm{M}$, and $\mathrm{L}$ these respective values are $j_{d}(0)=1.9 \times 10^{7}, 2.0 \times 10^{7}$, and $1.7 \times 10^{7} \mathrm{~A} / \mathrm{cm}^{2}$, which are consistent with the values obtained earlier $\left(2.2 \times 10^{7}, 2.1 \times 10^{7}\right.$, and $\left.1.8 \times 10^{7} \mathrm{~A} / \mathrm{cm}^{2}\right)$ from the shifts in the resistive transitions near $T_{c}$ (Fig. 2 and Eq. 77.

Fig. 1 shows the values of $I_{d}$ at different temperatures obtained from the $I V$ characteristics of Fig. 3(a). Also 


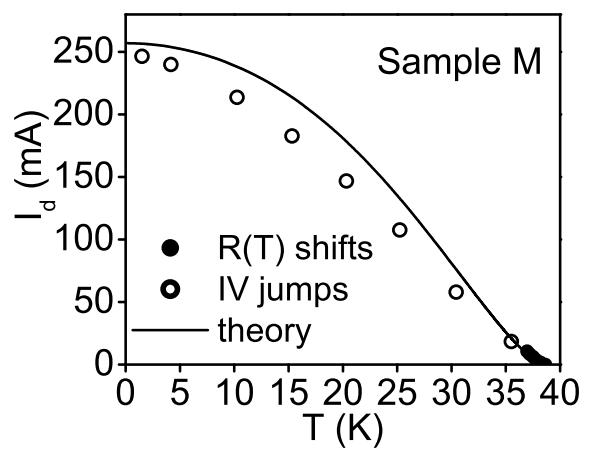

FIG. 4: Pair-breaking currents from IV curves (Fig. 3) and $T_{c}$ shifts (Figs. 1 and ). The solid line represents the theoretical curve (Eq. 5) in which $I_{d}(0)$ and $T_{c}$ are fixed by the $T_{c}$ shifts (Fig. . 2; $36<T<39$ ), with no adjustment made over the rest of the range $(1.5 \leq T \leq 36 \mathrm{~K})$.

shown are the values of $I_{d}$ obtained from the shifts in the resistive transition near $T_{c}$ (from Fig. 2). The solid line is a plot of Eq. 5 in which the values of $T_{c}(0)$ and $I_{d}(0)$ came directly from the observed $j^{2 / 3}$ behavior of Fig. 2 and were not adjusted to fit the other data over the extended temperature range, nor was $I_{d}(0)$ adjusted to fit the actual measured value from the $I V$ characteristic at low $T$ (i.e., Fig. 3). Nevertheless, the $I_{d}(T)$ data tend to follow the general trend of Eq. 5 .

An aspect of these data that may seem surprising is that even for $T \ll T_{c}$ (where the sample width $w \gg$ $\lambda, \xi)$, the average current density in the bridge reaches essentially the full $j_{d}$ before the system becomes normal. By contrast, previous studies 10 of $j_{d}$ in wide low- $T_{c}$ type-I bridges found that the current distribution was non-uniform and the sample was driven normal when the peak $j$ near the surface exceeded $j_{d}$. Using their model with our sample dimensions and parameters, our effective $j_{d}$ should have been reduced by a factor of 3 , but it is not. In our present relatively high- $T_{c}$ type II material, it seems that the slight flux motion induced by the self field and fluctuations (because of the proximity to the $T_{c}(j)$ phase boundary and much higher $T$ ) serve to homogenize the current distribution. Ironically, such incipient dissipation may actually stabilize the flow and permit the average $j$ to get closer to $j_{d}$ before the system becomes normal.

In conclusion, we have measured the fundamental pairbreaking critical current density of magnesium diboride over the entire temperature range for in-plane current transport. The measured $j_{d}(T)$ function is consistent with the Ginzburg-Landau form and conforms exactly to the $\Delta T_{c} \propto j^{2 / 3}$ behavior predicted near $T_{c} . \quad j_{d}(0)$ obtained from the value of current required to drive the sample normal at $T \rightarrow 0$, agrees with the $j_{d}(0)$ deduced from the $\Delta T_{c} \propto j^{2 / 3}$ behavior close to $T_{c}$. The average value for all samples by both methods is $j_{d}(0) \approx 1.9 \pm 0.4 \times 10^{7} \mathrm{~A} / \mathrm{cm}^{2}$. This is comparable in order of magnitude to the value of $6.1 \times 10^{7} \mathrm{~A} / \mathrm{cm}^{2}$ calculated from Eq. 6 and the published values of $H_{c}=2500$ $\mathrm{G}$ and $\lambda=185 \mathrm{~nm}$ from the review on $\mathrm{MgB}_{2}$ by Wang et al. [8], in view of the uncertainities in those parameters. From a technological standpoint, the depairing current density of $\mathrm{MgB}_{2}$ is about an order of magnitude lower than the high- $T_{c}$ cuprates [11. The good news is that the flux pinning in films is so strong (because of the larger coherence length and more isotropically 3-D behavior) that the depinning $j_{c}$ at modest fields appear to be within an order of magnitude of $j_{d}$ [12, whereas for the cuprates $j_{c}$ and $j_{d}$ can be separated by two or three orders of magnitude 13 .

\section{ACKNOWLEDGEMENTS}

The authors acknowledge useful discussions and other assistance from J. M. Knight, B. I. Ivlev, C. Wu, D. H. Arcos, H.H.Acros, H.J. Kim, E.M. Choi, K.J. Kim, and D. K. Finnemore. This work was supported by the U. S. Department of Energy through grant number DE-FG0299ER45763 and by the Creative Research Initiatives of the Korean Ministry of Science and Technology.

* URL: http://www.physics.sc.edu/kunchur; Electronic address: kunchur@sc.edu

[1] M. N. Kunchur, B. I. Ivlev, D. K. Christen, J. M. Phillips, Phys. Rev. Lett. 84, 5204 (2000).

[2] Michael Tinkham, Introduction to Superconductivity, 2nd Edition (McGraw Hill, New York, 1996).

[3] J. Bardeen, Rev. Mod. Phys. 34, 667 (1962).

[4] W. N. Kang, H.-J. Kim, E.-M. Choi, C. U. Jung, S.-I. Lee, Science 292, 1521 (2001); (10.1126/science. 1060822).

[5] W. N. Kang, E.-M. Choi, H.-J. Kim, H.-J. Kim, and S.-I. Lee, Preprint: cond-mat/0209226 at xxx.lanl.gov (2002).

[6] M. N. Kunchur, Phys. Rev. Lett. 89, 137005 (2002).

[7] M. N. Kunchur, Mod. Phys. Lett. B. 9, 399 (1995).

[8] Y. Wang, T. Plackowski, A. Junod, Physica C 355, 179 (2001).

[9] O. M. Stoll, S. Kaiser, R. P. Huebener, and M. Naito, Phys. Rev. Lett. 81, 2994 (2001).

[10] W. J. Skocpol, Phys. Rev. B 14, 1045 (1975).

[11] M. N. Kunchur, D. K. Christen, C. E. Klabunde, and J. M. Phillips, Phys. Rev. Lett. 72, 752 (1994).

[12] H.-J. Kim et al., Phys. Rev. Lett. 87, 87002 (2001); S. H. Moon et al., Appl. Phys. Lett. 79, 2429 (2001); and C. B. Eom et al. Nature 411, 558 (2001).

[13] Preliminary in-field measurements on $\mathrm{MgB}_{2}$ at low temperatures indicate $I V$ curves that are highly non-linear with no Ohmic free-flux-flow behavior at all. Instead the resistivity rises rapidly from its onset to its full normalstate value due to a combination of pair breaking and flux motion. Details of this non-linear flux flow behavior will be discussed elsewhere. 